\title{
Estudio de superficies de magnesio modificadas con fosfatos y películas poliméricas para aplicaciones médicas
}

\section{Study of magnesium surfaces modified with phosphates and polymeric films for medical applications} GARCIA-NAVARRO, Norma†*, GALICIA-AGUILAR, Gonzalo, LUGO-ISLAS, Gabriela y
GALLARDO-CASTÁN, Ernesto

Universidad Veracruzana, Campus Poza Rica Tuxpan

ID $1^{\text {er }}$ Autor: Norma, Garcia-Navarro / ORC ID: 0000-0002-0381-6617, Researcher ID Thomson: G-9585-2018, CVU CONACYT ID: 49611

ID $1^{\text {er }}$ Coautor: Gonzalo, Galicia-Aguilar / ORC ID: 0000-0002-0633-3442, Researcher ID Thomson: G-9497-2018, CVU CONACYT ID: 38494

ID $2^{\text {do }}$ Coautor: Gabriela, Lugo-Islas / ORC ID: 0000-0003-2572-3697, Researcher ID Thomson: G-9483-2018, CVU CONACYT ID: CVU -445987

ID $3^{\text {er }}$ Coautor: Ernesto, Gallardo-Castán / ORC ID: 0000-0002-4117-2891, Researcher ID Thomson: G-9590-2018, CVU CONACYT ID: 818820

DOI: $10.35429 / J T E N .2019 .12 .3 .8 .15$

Recibido 14 de Octubre, 2019, Aceptado, 07 de Diciembre, 2019

\begin{abstract}
Resumen
El magnesio y sus aleaciones han sido de interés científico en la regeneración ósea debido a sus características reabsorbibles, biocompatibles y mecánicas. Sin embargo, su actividad electroquímica es un reto, se buscan tratamientos de modificación superficial mediante biopolímeros o tratamientos de conversión para disminuir la velocidad de corrosión. El objetivo de esta investigación ha sido evaluar con técnicas electroquímicas el comportamiento corrosivo de magnesio en solución fisiológica simulada a través de una interfase de fosfatado y de una película de quitosano-grenetina. Se realizó el tratamiento de fosfatado por conversión química pH 10, 11 y 12 y se elaboró una película por solución polimérica y secado convectivo. Las superficies fueron caracterizadas por DRX, RAMAN, SEM-EDS. Se obtuvieron fases combinadas de fosfatos con morfología rugosa y porosa con propiedades no conductoras para relacionarse con la respuesta electroquímica de la interfase fosfatada con diferentes áreas de magnesio puro, estimándose el área fosfatada real. La película biopolimérica induce al proceso de corrosión a una velocidad de aproximadamente una cuarta parte de magnesio fosfatado durante 4 semanas de exposición en el medio fisiológico. Mejorar las propiedades de adhesión de la película biopolimérica y la porosidad podrían coadyuvar en la regeneración ósea durante este proceso corrosivo.
\end{abstract}

Magnesio, Corrosión, Polímeros

\begin{abstract}
Magnesium and its alloys have been of scientific interest in bone regeneration due to its resorbable, biocompatible and mechanical characteristics. However, its electrochemical activity is a challenge; surface modification treatments are sought through biopolymers or conversion treatments to reduce its corrosion rate. The objective of this research was to evaluate the corrosive behavior of magnesium in simulated physiological solution through electrochemical techniques through a phosphating interface and a chitosan-grenetine film. The phosphating treatment was carried out by chemical conversion $\mathrm{pH} 10,11$ and 12 and a film was prepared by polymer solution and convective drying. The surfaces were characterized by DRX, RAMAN, SEM-EDS. Combined phases of phosphates with rugose and porous morphology with non-conductive properties were obtained to relate to the electrochemical response of the phosphate interface with different areas of pure magnesium, estimating the actual phosphated area. The biopolymeric film induces the corrosion process at a rate of approximately a quarter of magnesium phosphate during 4 weeks of exposure in the physiological medium. Improving the adhesion properties of the biopolymer film and the porosity could contribute to bone regeneration during this corrosive process.
\end{abstract}

Magnesium, Corrosion, Polymers

Citación: GARCIA-NAVARRO, Norma, GALICIA-AGUILAR, Gonzalo, LUGO-ISLAS, Gabriela y GALLARDOCASTÁN, Ernesto. Estudio de superficies de magnesio modificadas con fosfatos y películas poliméricas para aplicaciones médicas. Revista de Ingeniería Tecnológica. 2019 3-12: 8-15

\footnotetext{
* Correspondencia del Autor (Correo electrónico: gnnorma@gmail.com)

$\dagger$ Investigador contribuyendo como primer autor.
} 


\section{Introducción}

El uso de materiales metálicos como implantes médicos ha contribuido en tratamientos $y$ reemplazo de estructuras óseas, principalmente por sus propiedades mecánicas. Sin embargo, se requiere que cumplan con una función específica temporal o permanente sin causar reacciones adversas en el organismo. Además, los implantes metálicos pueden fallar debido a la transferencia o cambios de carga, crecimiento de la estructura ósea o por corrosión generada por procesos metalúrgicos, geometría de diseño, entre otros.

Existen aleaciones como los aceros inoxidables al molibdeno que son utilizados en implantes con fines médicos, su desventaja se encuentra en que se debe recurrir a una segunda cirugía para lograr remover el implante una vez que ha cumplido su función en el cuerpo humano, además de las especies químicas tóxicas que puede liberar al contacto con los fluidos corporales propio del mecanismo de oxidación. Por tal motivo, la ciencia ha puesto su empeño en el estudio del Magnesio y sus aleaciones debido a la cinética de disolución rápida (corrosión) y su fácil degradación en el cuerpo humano. Es decir, si se logra controlar la velocidad de corrosión del magnesio, se podría utilizar en la medicina, desintegrándose totalmente una vez que haya cumplido su función y evitando así una segunda cirugía. El presente artículo contribuye en la búsqueda de métodos que permitan controlar la velocidad de corrosión del Magnesio.

Ante este panorama, la investigación multidisciplinaria se ha enfocado en utilizar diversos métodos de protección como son los tratamientos químicos con o sin plasma, electroquímicos, sputtering, entre otros, aislándolos del medio fisiológico para evitar la corrosión. Por otra parte, actualmente los biomateriales reabsorbibles por el organismo como son los biopolímeros, entre ellos quitosano (QS) y grenetina (GR) han sido de interés científico por sus características degradables y cumplimiento de los requerimientos de aplicación médica.

El magnesio $(\mathrm{Mg})$ ha sido una alternativa de aplicación, sin embargo, se ve limitada por su alta actividad electroquímica y evolución de $\mathrm{H}_{2}$ convirtiéndose en una problemática y reto si se pretendiera evitar el fenómeno corrosivo.
Desde una perspectiva más amplia, podría convertirse en una ventaja, debido a que el magnesio es necesario para las reacciones metabólicas del organismo y la formación de los huesos siempre que sea en cantidades asimilables por el organismo.

En este trabajo se integra la modificación superficial de $\mathrm{Mg}$ a través del tratamiento químico de fosfatado para el control de la corrosión y la elaboración de la película biopolimérica, se estudia el proceso de formación y comportamiento de la interfase con el medio fisiológico Kokubo.

\section{Metodología}

Se empleó como reactivos quitosano (99.9\%) desacetilado de peso medio, grenetina comercial, ácido cítrico $\mathrm{C}_{6} \mathrm{H}_{8} \mathrm{O}_{7}(99.8 \%)$, ácido fosfórico $\mathrm{H}_{3} \mathrm{PO}_{4}(86 \%)$, hidróxido de sodio $\mathrm{NaOH}$ (99\%), 2-Propanol $\mathrm{C}_{3} \mathrm{H}_{8} \mathrm{O}$, y solución fisiológica Kokubo. En cuanto a los materiales se usó magnesio (99.7\%), aire comprimido, una celda electroquímica cilíndrica de polimetilmetacrilato (PMMA) de dos compartimentos de $200 \mathrm{~mL}$ y otra de $400 \mathrm{~mL}$. Los electrodos fueron de grafito $(15 \mathrm{~cm}$ largo, $0.6 \mathrm{~cm}$ diámetro) y de $\mathrm{Ag} / \mathrm{AgCl}$ saturado, ColePalmer. Para la comprensión de la interfase de la Película QS-GR / Mg con Tratamiento Químico de Fosfatado (TQ) se realizó la evaluación electroquímica mediante técnicas de corriente alterna y corriente directa (con potenciostatos Gill AC ACM Instruments y Bio-Logic Science Instruments SP-150). Para ello fue necesario partir del análisis de magnesio puro o sin tratamiento. La caracterización se realizó por microscopía electrónica de barrido-análisis elemental FESEM, JEOL JSM-7600F, difracción de Rayos X (XRD), Bruker D8 Advance, espectroscopía RAMAN Scientific DRX-RAMAN y actividad de agua, AQUALAB.

La preparación superficial de las muestras de $\mathrm{Mg}$ se realizó con base a la metodología [1], realizando 1) un enjuague con agua destilada, baño ultrasónico con 2-propanol (15 min), secado con aire comprimido, 2) un enjuague con agua destilada y secado manual a temperatura ambiente, 3) enjuague y baño ultrasónico con agua destilada, secado con acetona y aire comprimido, 4) enjuague con agua destilada, secado con aire comprimido y exposición al ambiente $(1 \mathrm{~h})$. 
El arreglo del sistema electroquímico consistió en una celda rectangular cerrada de tres electrodos: $\mathrm{Ag} / \mathrm{AgCl}$ saturado como electrodo de referencia (ER), grafito como contra electrodo (CE) y magnesio puro como electrodo de trabajo (ET) con áreas de exposición 0.78 y $1.32 \mathrm{~cm}^{2}$. Se utilizó como electrolito solución fisiológica Kokubo SBF. Los volúmenes del electrolito fueron de $125 \mathrm{ml}$ para área $0.78 \mathrm{~cm}^{2}$ y $200 \mathrm{ml}$ para $1.32 \mathrm{~cm}^{2}$.

Las pruebas electroquímicas realizadas fueron: 1) Resistencia a la Polarización Lineal (LRP): Se realizó en función del tiempo (3 h 30 min) de $\pm 15 \mathrm{mV} /$ Ecorr y $0.16 \mathrm{mV} / \mathrm{s}$ de acuerdo a la NOM ASTM G59-97, considerándose intervalos de 10 minutos de estabilización $\mathrm{E}$ vs $\mathrm{t}$ entre cada prueba asegurando se mantenga el potencial estable, 2) Espectroscopía de Impedancia Electroquímica (EIS): $\mathrm{Se}$ consideraron distintas áreas para determinar la tendencia de la respuesta de EIS y estimar áreas sin fosfatar.

Se aplicó un intervalo de frecuencias de $10-30 \mathrm{kHz}$ a $10 \mathrm{mHz}$ con una amplitud de 10 $\mathrm{mV} / \mathrm{rms}$ y 6 puntos por década, 3) Extrapolación de Tafel: Se realizó al finalizar la prueba de Espectroscopía de Impedancia Electroquímica y posterior a 10 minutos de estabilización $\mathrm{E}$ vs t. Se aplicó un potencial de -120 a $300 \mathrm{mV}$ ya que a este a potencial se determinó el comportamiento de la pendiente anódica de interés para comparación con el sustrato con tratamiento químico y película polimérica. La velocidad de barrido $\mathrm{Vb}$ fue de $10 \mathrm{mV} / \mathrm{min}$ a Ecorr de acuerdo con la NOM G5-94.

Con los datos obtenidos de las evaluaciones de DC y AC se realizaron gráficas utilizando el programa OriginPro8. El análisis de datos a través de circuitos equivalentes se realizó en el software Boukamp.

La solución fosfatante se realizó por inmersión en una solución de $\mathrm{NaOH} 1 \mathrm{M}-\mathrm{H}_{3} \mathrm{PO}_{4}$ a diferentes pH's (10, 11 y 12). El tratamiento químico se realizó por inmersión de 24 horas, 5 días y 1 mes, durante este tiempo se mantuvo cubierto el vaso de precipitado a temperatura 25 ${ }^{\circ} \mathrm{C} \pm 5{ }^{\circ} \mathrm{C}$.

Las soluciones de biopolímeros se elaboraron con base a la metodología mencionada por Cárcamo, (2005).
Se consideraron concentraciones 1 y $2 \%$ $\mathrm{p} / \mathrm{v}$ (biopolímero/Ac.Cítrico) preparando por separado las soluciones de grenetina: quitosano en agua destilada a diferentes proporciones (1:1, 0.5:1, 0.6:0.4) por agitación magnética a $4 \mathrm{rpm}$ y $60{ }^{\circ} \mathrm{C}$ hasta solubilizar, posteriormente se mezclaron ambas soluciones. La solución de Ác. Cítrico $1 \%$ se adicionó por goteo a la solución biopolimérica manteniendo la agitación. La solución final se vertió en cajas de petri de plástico a volúmenes $10,18,25,36,50 \mathrm{~mL}$, para seleccionar la de mejor desprendimiento y manipulación. El tiempo de secado se varió entre 8 y 36 horas debido a los volúmenes mencionados.

Se ajustó el pH de las películas seleccionadas 6 por inmersión en solución de $\mathrm{NaOH} 0.1 \mathrm{M}$ durante 10 minutos, enjuagando 3 veces con agua destilada. Las muestras se secaron durante 20 minutos a $60^{\circ} \mathrm{C}$.

Se utilizó el arreglo del sistema electroquímico de $\mathrm{Mg}$ desnudo con área de exposición $1.76 \mathrm{~cm}^{2}$, se seleccionó una muestra de la película QS-GR de $2 \mathrm{~cm}^{2}$, sobreponiéndola a la muestra de Mg-TQ pH 10, 11 y 12, Figura 1. El volumen de electrolito, solución fisiológica Kokubo fue $350 \mathrm{~mL}$.

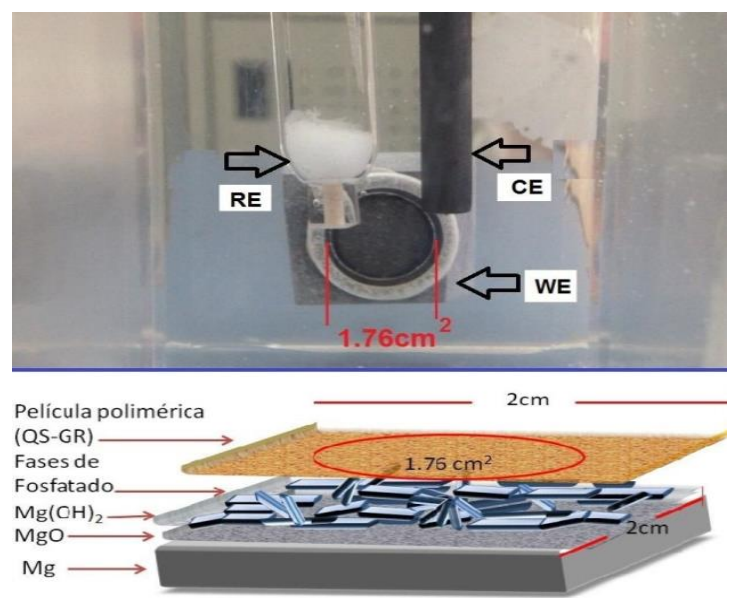

Figura 1 Arreglo electroquímico de Película QS-GR / MgTQ. Se asume la formación de $\mathrm{MgO}$ previa al tratamiento químico y la formación de $\mathrm{Mg}(\mathrm{OH})_{2}$ posterior a la inmersión en solución Kokubo.

\section{Resultados}

En la Figura 2 se presenta el proceso de estabilización durante 24 horas. De acuerdo con la teoría electroquímica, la Vcorr tiende a disminuir progresivamente durante el proceso de estabilización, dependiendo de la naturaleza de los productos formados. 
Considerando el desplazamiento del Ecorr hacia valores menos electronegativos se asume la formación de óxidos poco protectores en la superficie de $\mathrm{Mg}$, señalados en la literatura como $\mathrm{MgO}$ y $\mathrm{Mg}(\mathrm{OH})_{2}$ [2-6].

Las oscilaciones en la corriente mostradas en la Tabla 1, con alrededor de 30 a 120 minutos de exposición, se atribuyen a la interacción del ión $\mathrm{Cl}^{-1}$ con la capa de $\mathrm{Mg}(\mathrm{OH})_{2}$ para formar $\mathrm{MgCl}_{2}$, de carácter muy soluble [3]. Por otra parte, se observa que en función del tiempo de exposición hay un desplazamiento del Ecorr hacia valores menos electronegativos manteniéndose pequeñas oscilaciones de la velocidad de corrosión a mayor tiempo de estabilización [4].



Figura 2 Diagrama de estabilización del E vs t de Mg puro inmerso en $200 \mathrm{~mL}$ de solución Kokubo durante 24 horas

\begin{tabular}{|r|r|r|r|}
\hline $\begin{array}{c}\text { Tiempo } \\
(\mathrm{min})\end{array}$ & $\begin{array}{c}\text { Ecorr }(\mathrm{mV}) \\
\text { vs }\end{array}$ & \multicolumn{1}{c|}{$\begin{array}{c}\mathrm{Rp} \\
\left(\Omega . \mathrm{cm}^{2}\right)\end{array}$} & $\begin{array}{c}\text { Velocidad } \\
\text { Corrosión } \\
(\mathrm{mm} / \mathrm{año})\end{array}$ \\
\hline 0 & -1837 & 369 & 1.22 \\
\hline 30 & -1791 & 600 & 0.75 \\
\hline 60 & -1790 & 589 & 0.76 \\
\hline 90 & -1789 & 619 & 0.73 \\
\hline 120 & -1787 & 563 & 0.80 \\
\hline 150 & -1775 & 545 & 0.82 \\
\hline 180 & -1774 & 606 & 0.74 \\
\hline Promedio & -1792 & 556 & 0.83 \\
\hline
\end{tabular}

Tabla 1 Velocidades y potenciales de corrosión de Mg puro en solución Kokubo con estabilización de 24 horas. Área de exposición $1.32 \mathrm{~cm}^{2}$

La Figura 3 muestra la degradación de la interfase de puro en solución fisiológica Kokubo posterior al tiempo de estabilización y evaluación electroquímica. Es evidente que a menor tiempo de estabilización (1 hora) la muestra (b) presenta menor degradación comparada con la muestra (c) y (d), de 24 horas de estabilización.

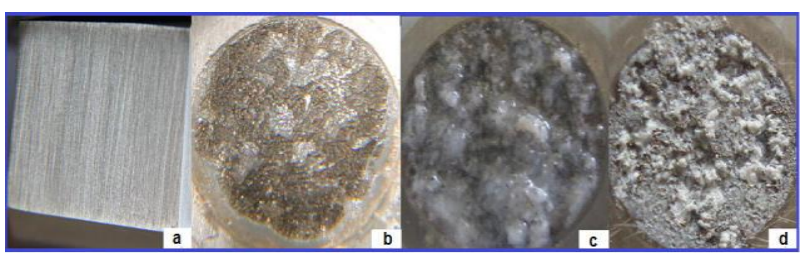

Figura 3 Superficies de $\mathrm{Mg}$ puro evaluadas electroquímicamente: (a) previa a la inmersión, (b) 1 hora de estabilización, (c) y (d) muestra con 24 horas de estabilización en solución Kokubo, antes y después de enjuagar con agua destilada respectivamente.

La evidencia de que el tipo de corrosión filiforme puede ser ocasionado por la extensión de grietas formadas a partir de la interacción de los iones $\mathrm{Cl}^{-}$con la capa de $\mathrm{Mg}(\mathrm{OH})_{2}$ )se observa en la micrografía SEM y análisis EDS de la Figura 4. Este tipo de corrosión es sugerido debido al incremento del $\mathrm{pH}$ local por hidrólisis de $\mathrm{Mg}^{+2}$, como un factor restrictivo para la corrosión localizada [5] el cual se contrapone a la corrosión por picadura mencionada en la literatura [6-9]. La presencia de $\mathrm{Ca}$ puede ser atribuida al agua destilada utilizada en el enjuague de la muestra.

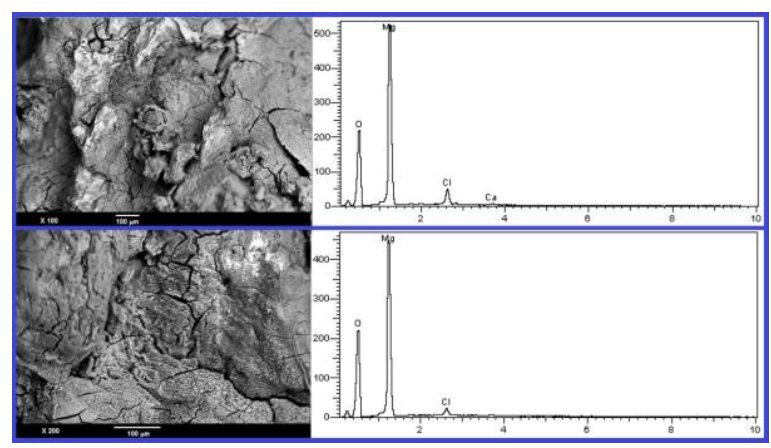

Figura 4 Micrografías SEM Mg desnudo expuesto en solución fisiológica-Análisis EDS Elemental

El análisis de la evolución de la interfase con estabilización de 1 y 24 horas se realizó mediante EIS a Ecorr.

Para la interfase de 24 horas, las gráficas de Nyquist, Figura 5, se caracterizan por presentar un semicírculo capacitivo en el intervalo de altas frecuencias y en el intervalo de medianas a bajas frecuencias $(1.36 \mathrm{~Hz}$ a $\approx 10$ $\mathrm{mHz}$ ) se observa un segundo semicírculo que de acuerdo con la literatura corresponden a la transferencia de carga y al transporte de materia respectivamente $[10,12]$.

Un tercer semicírculo de tipo inductor se presenta a muy bajas frecuencias $(f<10 \mathrm{mHz})$, atribuido a la adsorción de $M g_{a d s}^{+}$. 
Se observa el mismo efecto encontrado en LPR debido a la presencia de los iones $\mathrm{Cl}^{-}$, los cuales son responsables de que el sistema salga del equilibrio en la parte de bajas frecuencias, cuando $f \rightarrow 0, C A \cong C D$, afectando la formación definida del segundo semicírculo. En función del tiempo la resistencia tanto del transporte de carga como de materia disminuyen, reflejándose en el decremento de los semicírculos capacitivos, atribuido al deterioro de los productos de corrosión (formados durante el tiempo de estabilización) por interacción con los iones $\mathrm{Cl}^{-}$, que también pueden verse afectados por la perturbación a bajas frecuencias $(10 \mathrm{mHz})$, como se señaló anteriormente.

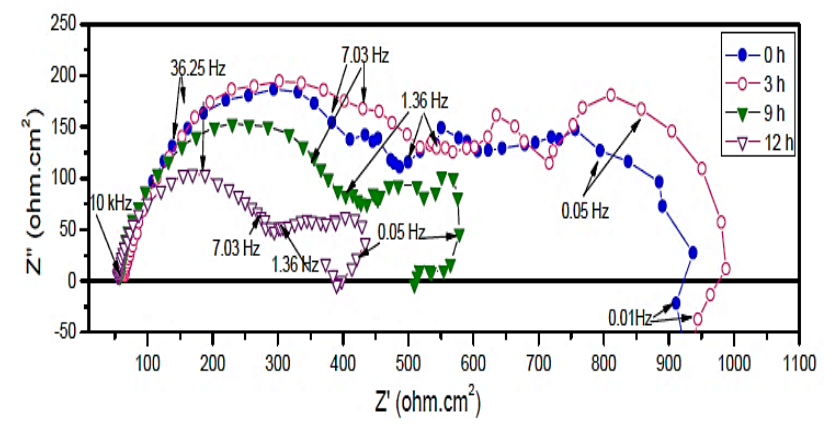

Figura 5 Diagrama de Nyquist de Mg puro posterior a 24 $\mathrm{h}$ de estabilización en solución Kokubo

Las micrografías SEM de las muestras a pH 10 y 11, Figura 6 describen una combinación de fases, señaladas en la literatura como estructuras cristalinas ortorrómbicas, trigonales, triclínicas y en forma de flor con una distribución no uniforme para ambas [13-16].

Se observa para pH 10 áreas sin formación de fases, sin embargo, para pH 11 la morfología se manifiesta más compacta. En las muestras de pH12 (inciso c) se observa una morfología que puede indicar la formación de una sola fase, descrita en la literatura como ortorrómbica, con una distribución no uniforme similar a pH10.

En las tres muestras se observa en el análisis EDS la presencia de fósforo $(P)$, magnesio $(M g)$ y oxígeno $(O)$ correspondientes al fosfatado, la presencia de $O$ puede incluso atribuirse a la formación de óxidos. Por otra parte, la presencia de sodio $(\mathrm{Na})$ se atribuye a la formación de precipitados ya que se utilizó $\mathrm{NaOH}$ durante el ajuste de $\mathrm{pH}$, el Ca es atribuido al agua destilada utilizada.

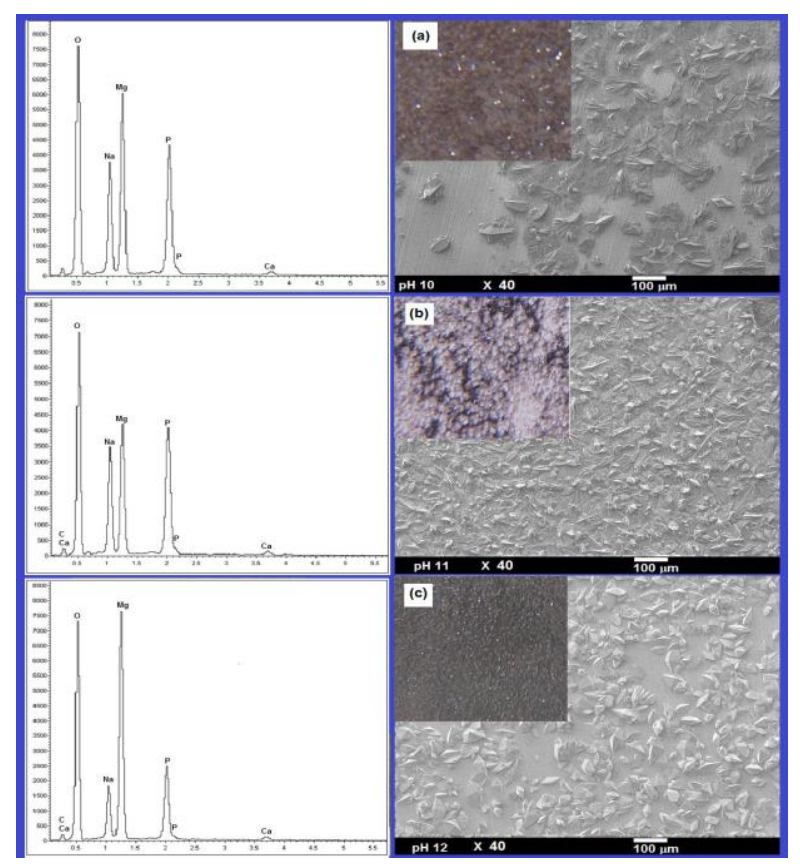

Figura 6 Muestras de Mg-TQ: (a) pH10, (b) pH11 y (c) pH12 e imágenes SEM-EDS a X40

En la caracterización por XRD, Figura 7, se identificaron para $\mathrm{pH} 10$ y 11 a $1^{\circ}$ y 1 segundo (s) de exposición fases de fosfatos hidratadas, correspondientes a $\mathrm{Mg}\left(\mathrm{HPO}_{4}\right)_{2}, \mathrm{Mg}\left(\mathrm{H}_{2} \mathrm{PO}_{4}\right)_{2}$ $\mathrm{Mg}_{3}\left(\mathrm{PO}_{4}\right)_{2}, \mathrm{Mg}_{3}\left(\mathrm{PO}_{4}\right)_{2} \cdot 4 \mathrm{H}_{2} \mathrm{O}$ y $\mathrm{Mg}$. Para $\mathrm{pH} 12$, fue necesario disminuir el ángulo de incidencia a $0.5^{\circ}$ y aumentar el tiempo de exposición a $10 \mathrm{~s}$, (Figura 8). Se observa la formación de fases de $\mathrm{Mg}_{3}\left(\mathrm{PO}_{4}\right)_{2}, \mathrm{Mg}_{3}\left(\mathrm{PO}_{4}\right)_{2} \cdot 4 \mathrm{H}_{2} \mathrm{O}$ y $\mathrm{MgO}$, y se corroboró la tendencia a formar una sola fase cuyo espesor se sugiere es inferior al pH 10 y 11 , además de observarse una mayor dispersión de la cristalinidad.

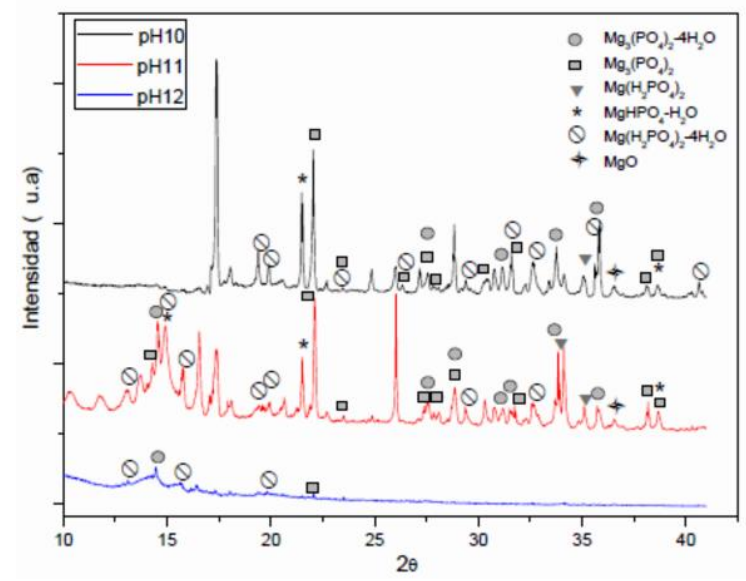

Figura 7 Difractograma de muestras de $\mathrm{Mg}$ fosfatado a $\mathrm{pH}$ 10,11 y 12 . Condiciones medición $1^{\circ}-1 \mathrm{~s}$ 


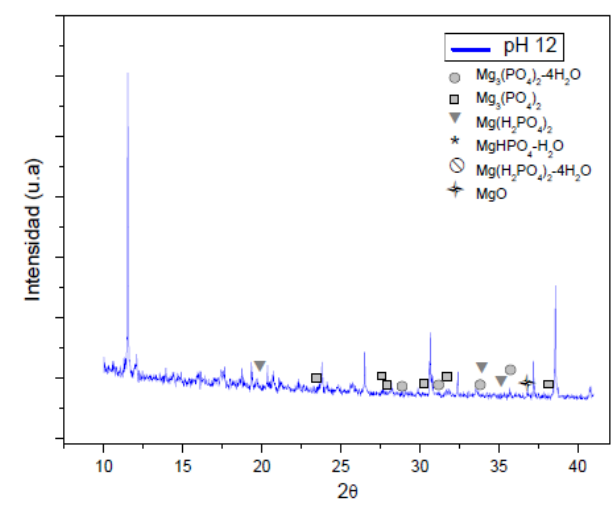

Figura 8 Difractograma de muestras de Mg Fosfatado pH12. Condiciones de medición $0.5^{\circ}-10 \mathrm{~s}$

\section{Películas de Quitosano-Grenetina QS-GR}

La formación de las películas se infiere por intercalación de quitosano-grenetina con ácido cítrico. De acuerdo con la literatura, el entrecruzamiento de estos polímeros es por interacción iónica de los grupos amino del quitosano y carboxílicos de la grenetina, el ácido cítrico ha sido empleado como agente entrecruzante y disolvente del quitosano [6-17]. El análisis XRD, Figura 9, presenta la estructura semicristalina correspondiente a cada polímero con picos característicos próximos a $10^{\circ}, 20^{\circ}$ $[17,19]$. Comparados con los difractogramas de las películas $\mathrm{MF}(2 \% \mathrm{p} / \mathrm{v})$ y $\mathrm{MH}(1 \% \mathrm{p} / \mathrm{v})$, Figura 10, se observa mayor deformación para MF y únicamente para $\mathrm{MH}$ a $20^{\circ}$ del ángulo $2 \theta$ se mantiene un pico con mayor amplitud que el presentado en los polímeros puros; ambos resultados podrían corroborar la posible formación de la estructura amorfa por entrecruzamiento, como se ha mencionado es mayormente visible con el incremento de la concentración.

A mayor concentración biopolimérica se incrementan la intensidad de los grupos funcionales.

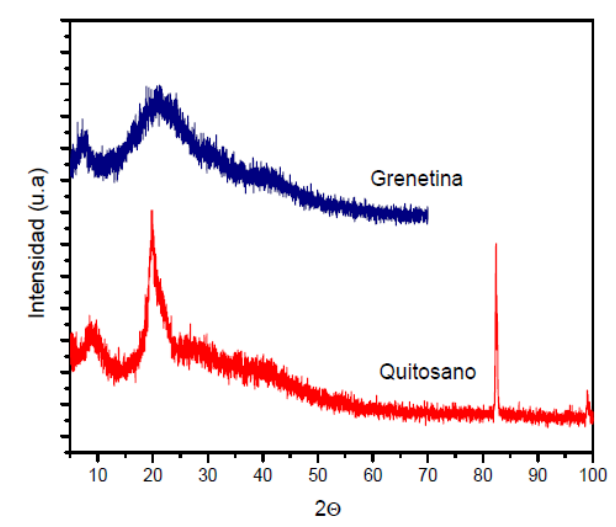

Figura 9 Difractograma de Quitosano peso molecular medio y grenetina comercial

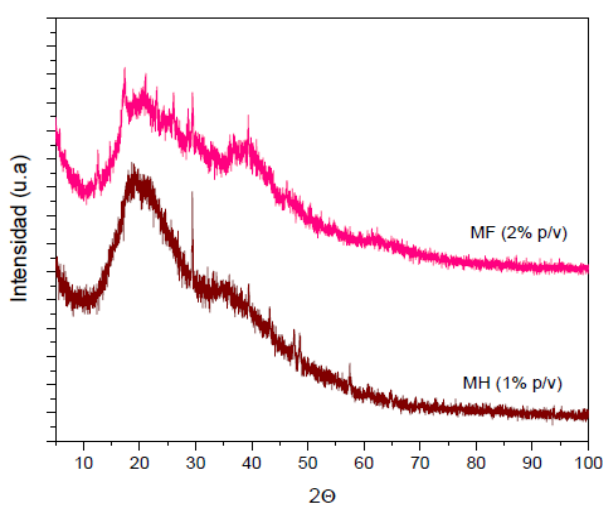

Figura 10 Difractograma de películas de QuitosanoGrenetina a concentración $1 \%$ y $2 \%$ p/v

Las micrografías de la Figura 11 describen una morfología fibrosa (a) de quitosano, que podrían dar lugar a porosidades y una morfología laminar (b) atribuida a la plasticidad de la grenetina, ambas asociadas a la deshidratación y deterioro de las redes poliméricas. Se visualiza que posterior a la rehidratación con $30 \mu \mathrm{L}$ de agua, $47.3 \%$ (a1) y $60 \%$ (b1), la recuperación de la película es próxima a las condiciones iniciales.

Por otra parte, durante el mes de inmersión en solución Kokubo, las películas presentan capacidad de absorción. Sin embargo, no recuperan el espesor de las condiciones iniciales, lo que indica que requiere de un porcentaje mínimo de humedad para mantener su estructura y al sobresaturarse describe un proceso de degradación.



Figura 11 Micrografías SEM de películas de QS-GR (1 y $2 \% \mathrm{p} / \mathrm{v}$ ): (a) y (b) películas deshidratas, (a1) y (b1) rehidratadas con $\mathrm{H}_{2} \mathrm{O}$ destilada, $(\mathrm{a} 1 *)$ y $(\mathrm{b} 1 *)$ espesor de las películas rehidratadas 
Asimismo, la muestra $\mathrm{MH}$ inmersa un mes en solución fisiológica, Figura 12, presenta un espesor inferior al inicial, sin embargo, el proceso de degradación anteriormente expuesto se hace evidente a X3000, con el desprendimiento de una capa ligera atribuible a la grenetina sobre la superficie grumosa de quitosano, por lo tanto, a X10 000 se visualiza la formación de porosidades. El análisis de superficies SEM, permitió conocer que la característica laminar de la grenetina confiere propiedades plásticas y obstruye la porosidad de la matriz de quitosano, por lo que representa una desventaja para el transporte de materia a través de ella inicialmente. A mayor tiempo de exposición la película se sobresatura y se propicia el proceso de degradación, evidenciándose con el análisis SEM la formación de porosidades.

El comportamiento hidrofílico de una de las caras de la película proporcionado por la grenetina mejora la porosidad e hinchamiento de ésta.

El comportamiento hidrofóbico proporcionado por quitosano se vio beneficiado al elevar el $\mathrm{pH}$ de la película.

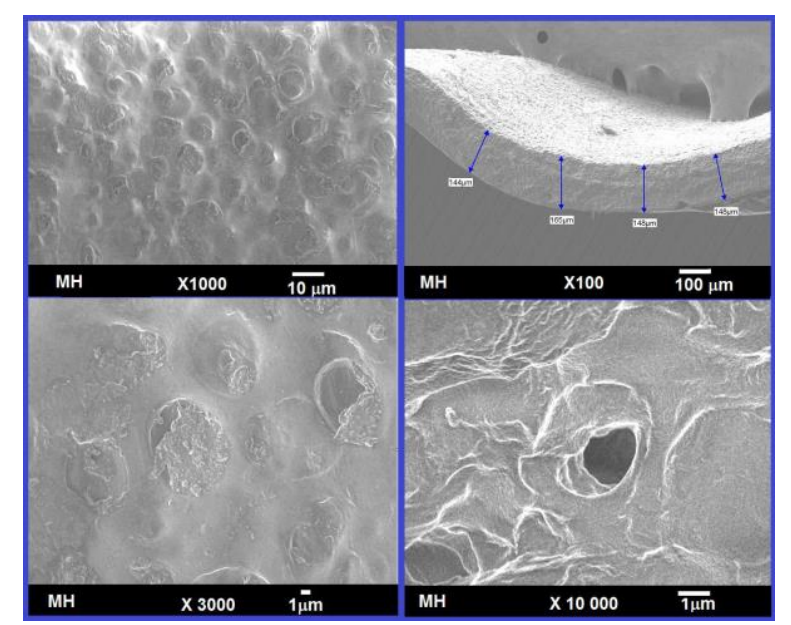

Figura 12 Micrografías SEM de películas de QS-GR MH $(1 \%)$ inmersa un mes en solución Kokubo.

\section{Conclusiones}

La presente investigación contribuye con mejoras para el desarrollo del tratamiento químico de fosfatado y aplicaciones biopoliméricas como un método de control de la velocidad de corrosión. Para ello, es relevante comprender el comportamiento individual de los materiales en el medio fisiológico Kokubo.
El tratamiento de fosfatado requiere del control del desbaste, secado y formación de óxidos de forma natural. La evolución de la interfase se limita la formación de fosfatos, debido a que el $\mathrm{pH}$ controla la formación de fosfatado, a pH10 y 11 se obtuvieron capas no uniformes de fases de fosfato combinadas, mayormente compactas para $\mathrm{pH} 11$. A pH12 se sugiere la obtención de una fase totalmente dispersa. Las características principales del fosfatado son no conductoras, de morfología rugosa y porosa.

La película polimérica de QuitosanoGrenetina (QS-GR) presentó características plásticas, de absorción y antimicrobiana. El carácter plástico obstruye la presencia de poros y limita la transferencia de materia a través de ésta. El carácter hidrofílico de una de las caras de la película promueve el hinchamiento de la matríz polimérica evitando el contacto con el sustrato metálico. La capacidad de absorción de la película indujo al comportamiento de un electrolito desde las primeras horas de exposición al medio fisiológico, esta misma capacidad promueve la degradación de quitosano y grenetina.

El fosfatado y su morfología, así como la película biopolimérica contribuyen en la respuesta electroquímica en los primeros instantes de exposición. El proceso corrosivo de la interfase película QS-GR/Mg-TQ pH10 fue iniciado por contacto de la película en la circunferencia de la superficie metálica expuesta. Por lo tanto, la velocidad de corrosión de la interfase es aproximadamente una cuarta parte de la presentada por Mg-TQ pH10 en los primeros instantes de exposición y se mantiene en el mismo orden de magnitud durante 4 semanas de exposición en el medio fisiológico, con tendencia a un control cinético anódico.

La contribución principal de la aplicación del tratamiento fosfatado de magnesio es que permitió correlacionar el área real fosfatada y la estimación de la velocidad de corrosión, por ende, se dedujo que es posible controlar el proceso corrosivo en un medio fisiológico simulado.

La desventaja presentada es que al no tener un control en la formación y distribución del fosfatado el desprendimiento del mismo es inmediato. 
La aplicación de la película biopolimérica no permite contribuir en el control del proceso corrosivo. Sin embargo, siempre que se conozca la porosidad y se asegure la adherencia de la misma o de los polímeros sobre el sustrato metálico, puede considerarse una alternativa de aplicación.

\section{Referencias}

[1] Ramírez, J. (2013). Tesis de maestría: Estudio del mecanismo de corrosión en un biomaterial base magnesio. México.

[2] Song, G., \& Atrens, A. (2017). Recent Insights into the Mechanism of Magnesium Corrosion and Research Suggestions. Advance Engineering Materials, 9(3), 177-183.

[3] Kirkland, N. T., Birbilis, N., \& Staiger, M. P. (2012). Assessing the corrosion of biodegradable magnesium implants: A critical review of current methologies and their limitations. Acta Biomaterialia, 8, 925-936.

[4] Atrens, A., Song, G. L., Cao, F., Shi, Z., \& Bowen, P. K. (2013). Advances in Mg corrosion and research suggestions. Journal of Magnesium and Alloys, 1, 177-200.

[5] Ma, W. H., Liu, Y. J., Wang, W., \& Zhang, Z. (2015). Improved biological performance of magnesium by micro-arcoxidation. Braz.J.Med.Biol.Res, 48(3), 214-225.

[6] Xue, D., Yun, Y., Tan, Z., Dong, Z., \& Schulz, M. (2012). In vivo and In Vitro Degradation Behavior of Magnesium Alloys as Biomaterials. J.Mater.Sci.Technol, 28(3), 261267.

[7] Li, N., Guo, Y., Wu, H., Zheng, Y. F., \& Ruan, L. Q. (2012). Comparative study on corrosion behaviour of pure Mg and WE43 alloy in static, stirring and flowing Hank's solution. Corrosion Engineering, Science and Technology, 47(5), 346-351.

[8] Galicia, G., Pébère, N., Tribollet, B., \& Vivier, V. (2016). Corrosion study of an AZ91 magnesium alloy by EIS and LEIS. ECS Transactions, 1(4), 157-168.

[9] Cao, F., Shi, Z., Hofstetter, J., Uggowitzer, P. J., Song, G., Liu, M., \& Atrens, A. (2013). Corrosion of ultra-high-purity $\mathrm{Mg}$ in $3.5 \% \mathrm{NaCl}$ solution satured with $\mathrm{Mg}(\mathrm{OH})_{2}$. Corrosion Science, 75, 78-99.
[10] Wu, C., Wen, Z., Dai, C., Lu, Y., \& Yang, F. (2010). Fabrication of calcium/chitosan coating on AZ91D magnesium alloy with a novel method. Surface \& Coating Technology, 294, 3336-3347.

[11] Tamimi, F., Nihouannen, D. L., Bassett, D. C., Ibasco, S., Gbureck, U., Knowles, J., . . . Barralet, J. E. (2011). Biocompatibility of magnesium phosphate minerals and their stability under physiological conditions. Acta Biomaterialia, 7, 2678-2685.

[12] Zhang, E. (2015). Phosphate treatment of magnesium alloy implants for biomedical applications. In Surface Modification of Magnesium and its Alloys for Biomedical Applications (pp. 23-57).

[13] Narayan, S., Song, I., \& Lee, M. (2015). Surface Modification of Magnesium and its Alloys for Biomedical Applications. Modification and Coating Techniques, 2.

[14] Chávez, A. (2013). Obtención depelículas de quitosano a partir de la quitina proveniente de desechos de la industria cangrejera. Universidad de Zulia- Maracaibo-Venezuela.

[15] Rangel, S., García, J., \& Orrego, C. (2011). Inmovilización de Lipasa de Cacndida antaretica sobre soportes de Quitosano-Gelatina. Rev.Colomn.Quím, 40(2), 149-164.

[16] Adar, F. (2012). Interpreting RamanSpectro of Functionalized Polymers. Applying the Tricks of the Trade. Spectroscopy, 27(6), 14-18.

[17] Sahoo, R., Sahoo, S., \& Lochan, P. (2013). Synthesis and characterization of ggelatinchitosan nanocomposite to explore the possible use as drug delivery vehicle. European Scientific Journal, 9(18) 